2005

\title{
Toward an automated dental identification system
}

Gamal Fahmy

Diaa Nassar

Eyad Haj-Said

Hong Chen

Omaima Nomir

See next page for additional authors

Follow this and additional works at: https://researchrepository.wvu.edu/faculty_publications

\section{Digital Commons Citation}

Fahmy, Gamal; Nassar, Diaa; Haj-Said, Eyad; Chen, Hong; Nomir, Omaima; Zhou, Jindan; Howell, Robert; Ammar, Hany; AbdelMottaleb, Mohamed; and Jain, Anil, "Toward an automated dental identification system" (2005). Faculty Scholarship. 411.

https://researchrepository.wvu.edu/faculty_publications/411 
Authors

Gamal Fahmy, Diaa Nassar, Eyad Haj-Said, Hong Chen, Omaima Nomir, Jindan Zhou, Robert Howell, Hany Ammar, Mohamed Abdel-Mottaleb, and Anil Jain 


\title{
Toward an automated dental identification system
}

\author{
Gamal Fahmy \\ Diaa Nassar \\ Eyad Haj-Said \\ West Virginia University \\ Department of Computer Science and Electrical Engineering \\ Morgantown, West Virginia 26506 \\ E-mail: fahmy@csee.wvu.edu \\ Hong Chen \\ Michigan State University \\ Computer Science and Engineering Department \\ East Lansing, Michigan 48823 \\ Omaima Nomir \\ Jindan Zhou \\ University of Miami \\ Electrical and Computer Engineering Department \\ Miami, Florida 33146 \\ Robert Howell \\ Hany H. Ammar \\ West Virginia University \\ Department of Computer Science and Electrical Engineering \\ Morgantown, West Virginia 26506 \\ Mohamed Abdel-Mottaleb \\ University of Miami \\ Electrical and Computer Engineering Department \\ Miami, Florida 33146 \\ Anil K. Jain \\ Michigan State University \\ Computer Science and Engineering Department \\ East Lansing, Michigan 48823
}

\begin{abstract}
Forensic odontology has long been carried out by forensic experts of law enforcement agencies for postmortem identification. We address the problem of developing an automated system for postmortem identification using dental records (dental radiographs). This automated dental identification system (ADIS) can be used by law enforcement agencies as well as military agencies throughout the United States to locate missing persons using databases of dental $x$ rays of human remains and dental scans of missing or wanted persons. Currently, this search and identification process is carried out manually, which makes it very time-consuming in mass disasters. We propose a novel architecture for ADIS, define the functionality of its components, and describe the techniques used in realizing these components. We also present the performance of each of these components using a database of dental images. () 2005 SPIE and IS\&T. [DOI: 10.1117/1.2135310]
\end{abstract}

\section{Introduction}

Law enforcement agencies have been exploiting biometric identifiers for decades as key tools in forensic identification. With the evolution of information technology and the huge volume of cases that must be investigated by forensic specialists, automation of forensic identification became inevitable. Forensic identification can take place prior to death and is referred to as antemortem (AM) identification. Identification can also be carried out after death and is

Paper 04130RR received Jul. 10, 2004; revised manuscript received Apr 7, 2005; accepted for publication Apr. 18, 2005; published online Dec. 1, 2005.

1017-9909/2005/14(4)/043018/13/\$22.00 @ 2005 SPIE and IS\&T. 
called postmortem (PM) identification. While behavioral characteristics (e.g., speech) are not suitable for PM identification, most of the physiological characteristics are not appropriate for PM identification. This is due to severe circumstances encountered in mass disasters (e.g., airplane crashers) or when identification is being attempted more than 2 weeks postmortem. Therefore, a postmortem biometric identifier must survive such severe conditions and resist early decay that affects body tissues. Dental features are considered the best candidates for PM identification due to their survivability and diversity. Forensic odontology is the branch of forensics concerned with identifying human individuals based on their dental features. Traditionally, forensic odontologists relied on the morphology of dental restorations (fillings, crowns, etc.) to identify victims. However, since modern materials used in restorations and fillings have poor radiographic characteristics and cavities in today's children and their children will be virtually unknown due to the advances in dentistry, ${ }^{1-3}$ it is becoming increasingly important to make identification decisions based on inherent dental features such as root and crown morphologies, tooth size, rotations, spacing between teeth, and sinus patterns rather than visual analysis of dental work (fillings, crowns, etc.).

Based on the information provided by experts from the Criminal Justice Information Services Division (CJIS) of the Federal Bureau of Investigation (FBI), there are over 100,000 unsolved missing person cases in the National Crime Information Center (NCIC) at any given point in time, $60 \%$ of which have remained in the computer system for 90 days or longer. These NCIC services are used nationwide by law enforcement agencies. In 1997, the CJIS of the FBI created a dental task force (DTF), whose goal is to improve the utilization and effectiveness of the NCIC missing and unidentified persons (MUP) files. ${ }^{4}$ The DTF recommended the creation of a digital image repository (DIR) and an automated dental identification system (ADIS). CJIS includes in its strategic plan the creation of an ADIS, with goals and objectives similar to the automated fingerprint identification system (AFIS) but using dental characteristics instead of fingerprints. ADIS will provide automated search and matching capabilities for digitized x-ray and photographic images. Hence, it will not only speed up the PM identification process but will also achieve more accurate and reliable results.

In this paper, we propose an architecture for ADIS and describe its components and functionalities. We highlight the techniques proposed to implement every component and give examples of their performance.

\section{Background}

Several computer-aided PM identification systems have been developed in the last two decades such as CAPMI (Ref. 5) and WinID (Ref. 6). Although these systems were mainly developed for identifying people in the military, they do not provide high level of automation, and feature extraction, coding, and image comparison are still carried out manually. Moreover, the dental codes used in these systems capture only artificial dental work. The NCIC has a dental database for missing persons and currently the search is still based on human coding of dental records into textual data.
ADIS is a process automation tool for PM identification that is being designed to achieve accurate and timely identification results with minimum amount of human intervention. To this end, ADIS will not only automate some of the steps taken by forensic experts to examine MUP cases, but it will also intelligently analyze radiographs to utilize the underlying image structures that are often difficult to be assessed merely by visual examination.

Image segmentation is typically defined as the process of extracting objects from the image background. It is usually performed by subtracting the object location from the rest of the image. This object location is usually calculated by an edge detection, an intensity measure or a target recognition algorithm. However, most of these techniques suffer from different types of noise due low resolution or poor lighting, which results in unsuccessful segmentation.

Segmentation subdivides an image into it's constitute regions or objects. In the dental image perspective, segmentation is to recognize and label individual tooth in the $\mathrm{x}$-ray image or parts of the tooth such as crown and root of the tooth. Each tooth or object extracted from the image represents region of interest (ROI) that contains important data used for later steps. The ROI is defined as a rectangular part of the image that focuses on one object of the extracted objects from the image. The resulting image represents an $\mathrm{x}$-ray image and the specified object inside the rectangle represents the ROI.

In most of the segmentation algorithms, the segmentation is done either by extracting region-based features that can identify different objects and regions, or by applying a model and try to adjust its parameters to fit the processed objects or regions. Although model-based approaches are more complicated, they are more successful and reliable. For a complete review of image segmentation see Ref. 7.

Enhancement of a dental radiograph is the process of producing an improved quality image out of a degraded quality input image of a dental radiograph. Here "higher quality" is a fuzzy term that requires further explanation. The quality of an image is a measure of its suitability for an application-specific manipulation. In ADIS, good-quality dental radiographs are those that would result in valid segments when used in conjunction with a suitable segmentation technique. Most segmentation techniques require high definition of object boundaries. From an image-processing point of view, a digitized dental radiograph is an 8-bit gray scale image (at least for the dental image database provided by the CJIS). The image size depends on the film type and the digitization resolution. Image resolution is characterized by the sampling rate (number of captured pixels per unit length) and the number of bits used in encoding the pixel colors (number of possible quantization levels). The CJIS database contains dental radiographs with various sampling rates. In Ref. 8, a comprehensive study about images enhancement and restoration is provided.

Mathematical morphology is a topological and geometrically based approach for image analysis. It is a powerful technique in extracting different shapes and structures in different applications such as texture classification, pattern analysis, and content-based image coding and retrieval. Morphological filtering is typically defined as grouping different pixels in the images based on their color, spatial frequency, and intensity. Objects in the morphologically 
processed image are usually well identified by a group of pixels that represent the objects shape. The main morphological filters used in shape reconstruction (grouping different objects) are erosion, dilation, opening, and closing. In Ref. 9, a complete review of morphological filtering is presented.

\section{ADIS Identification Functionality and Scenarios}

The ADIS functionality proposed in this paper consists of two processes, identify a subject and maintain the system. The process of identifying a subject is initiated when a user submits the subject's record (containing image and nonimage information). The result of identification should be a "short" list of few candidates whose reference records are retrieved from the DIR and presented to the forensic expert who makes a final decision about the identity of the subject MUP. The process of maintaining the system primarily would include updating the repositories (adding/purging) reference records and evaluating the performance of ADIS based on algorithms and techniques adopted in identification and matching.

In ADIS, we can use the dental codes that currently exist $^{10}$ in the NCIC, as an option to enhance the retrieval and matching speed and performance. We note here that these NCIC codes are manually extracted from MUP. Although ADIS is designed to be an automated system, it will also enable the use of the NCIC database for results refinements. To populate the DIR, high-level features are extracted from all the reference images and are stored in the DIR. ADIS will consist of two main components, the potential match component and the image comparison component, as presented in the next section.

When the user submits a subject record, that is typically an unidentified PM record, two processes occurs simultaneously:

1. NCIC codes are extracted from the subject image.

2. High-level features are extracted from the images, and these features are extracted through the potential matching component.

The NCIC-extracted codes can help in narrowing down the search list from an order of thousands to an order of hundreds. Note here that ADIS will be designed to function independently of the NCIC search. High-level features extracted from the dental records in the DIR would be available to a potential matching component. This potential matching component extracts high-level features to search the database and retrieve a potential match list for the query submitted record.

The image comparison component matches a pair of records and determines if there is a positive identification. Typically, the decision process is based on extraction, segmentation, and alignment and matching of feature points (a feature point is a generic notion that includes points, line segments, corners, etc.) from the records. The image comparison matching stage will produce a short list of positive matches. This short list (of the order of few images) is then given to the forensic expert who makes the final decision for positive identification.

Once a positive identification is made for a subject image, its corresponding reference image is removed from the
DIR. If a positive identification cannot be made for a subject image, then this image is added to the DIR as a new missing or unidentified image. In our system, if the subject record is AM, then the reference records are PM, and vice versa. However, we will also enable both subject and reference images to be AM to avoid database redundancy if an $\mathrm{AM}$ record of a missing person was submitted multiple times.

\section{Proposed ADIS Architecture}

In this section, we briefly describe the main three components in ADIS: the DIR component, the potential search matching component, and the image comparison component, as shown in Fig. 1.

\subsection{DIR}

The DIR is a central system that enables the voluntary submission, storage, and retrieval of digital images relating to missing and unidentified person investigations. The DIR will serve as an electronic centralized location where law enforcement agencies can submit digital images of dental $\mathrm{x}$ rays, and other information regarding MUP cases. It will eliminate the need to obtain $\mathrm{x}$ rays from other law enforcement agencies for identification purposes. In addition, it will help the forensic scientist to access the DIR to study the available images for quickly determining an exact match. The repository will contain image information as well as nonimage information for each missing/unidentified person. The procedure of DIR archiving is carried out after extracting dental features from each case's dental images. These dental features (which can all be extracted automatically) would include the shape and size of each tooth, the root curvature, if there were any extracted tooth, and the tooth spacing. The DIR would also contain nonimage information, such as case ID, if this record were for a PM or AM, the last time this record was updated.

We can use the DIR system for archiving and retrieval as follows:

\subsubsection{DIR archiving}

1. Reference record archiving request (nonimage information and images).

2. Archive the nonimage information.

3. For each of the submitted image, archive image information and extract features for each tooth.

4. Calculate the total number of teeth while archiving the whole available images.

\subsubsection{DIR retrieval}

1. Submit a subject image(s).

2. Extract features.

3. Search the DIR for highly matched records according to some similarity measures

4. List the final matched records' information.

\subsection{Potential Search Matching Component}

This component retrieves a list of candidate images (of the order of hundreds to tens) for every subject image from the DIR. In extracting the high-level features, two methodolo- 


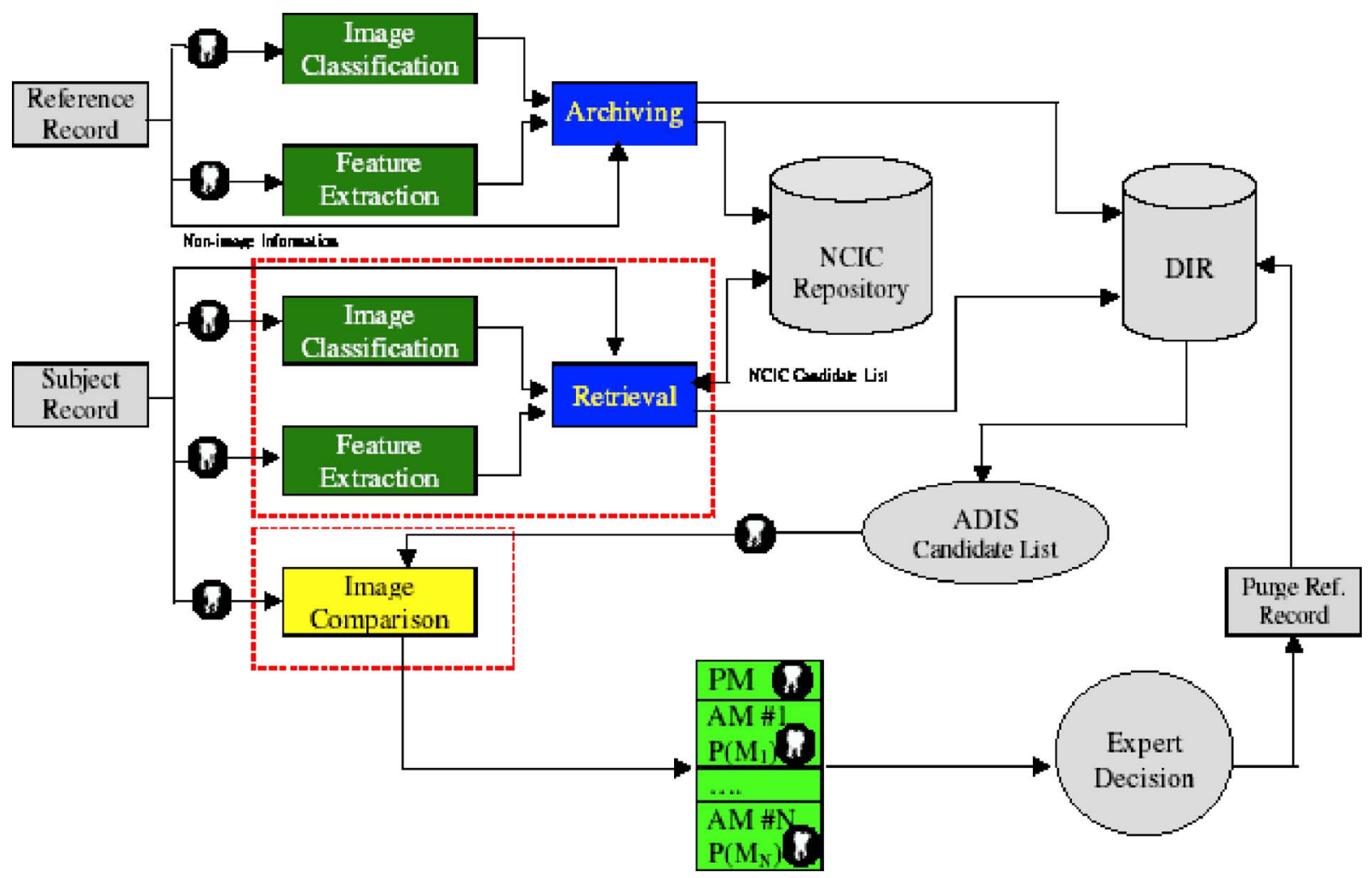

Fig. 1 Block Diagram of ADIS

gies are adopted in ADIS. The first methodology focuses on extracting tooth regions, whereas the second methodology focuses more on extracting the contour of roots and crowns, which are usually important in perapical images, as shown in Fig. 2, as well as bitewing images, as shown in Fig. 3.

\subsubsection{Contour extraction for bitewing images}

In this methodology, bitewing radiograph images are automatically enhanced and segmented. This methodology involves two main stages: bitewing enhancement and bitewing segmentation.

In the bitewing enhancement stage, dental radiographs contain three distinctive regions: background, teeth, and bones. Usually the tooth regions have the highest intensity, the bone regions have a high intensity that sometimes is close to that of the teeth, and the background has a distinc-
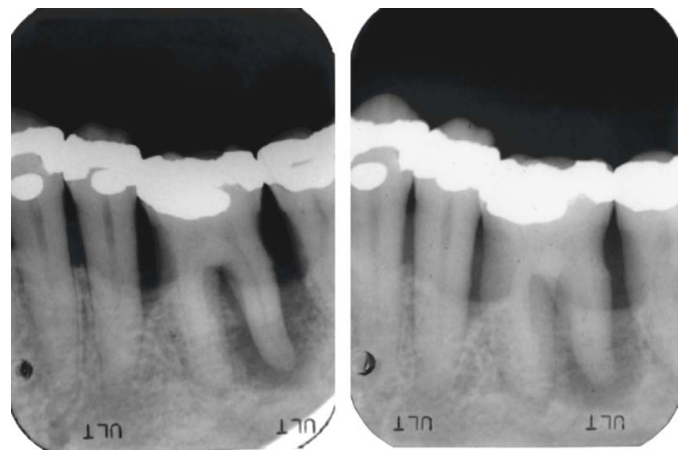

Fig. 2 Perapical dental images. tively low intensity. It is easy to separate the background by threshold-based methods, but these methods usually fail to discriminate teeth from bones, especially in cases of uneven exposure. To overcome this problem, the first step we use is to enhance the image's contrast. Top-hat and bottomhat morphological filters ${ }^{11}$ can be used to extract light objects (or, conversely, dark ones) on a dark (or light) but slowly changing background. We use both the top-hat and the bottom-hat filters on the original image, and combine the results by adding to the original image the result of the top-hat filter, and then subtracting the result of the bottomhat filter, so that the tooth areas can be enhanced and the

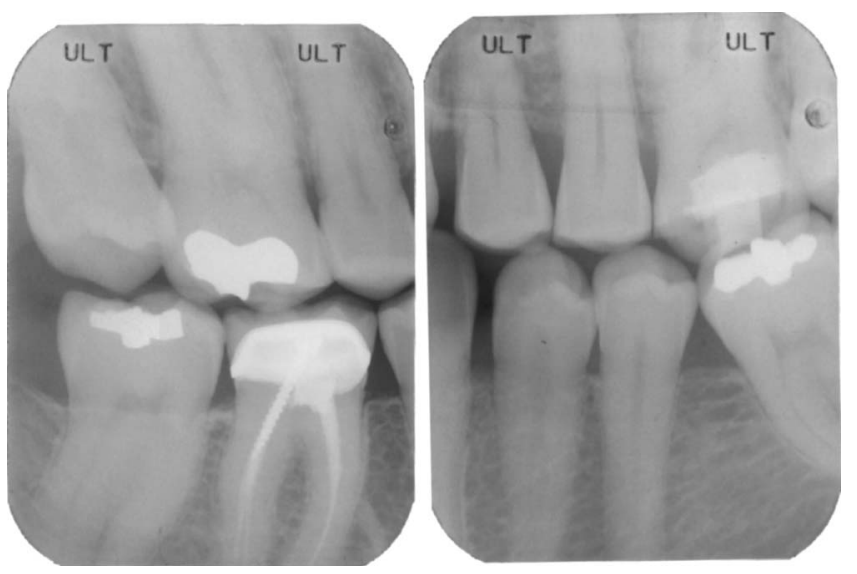

Fig. 3 Bitewing dental images. 


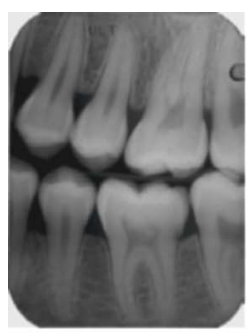

(a)

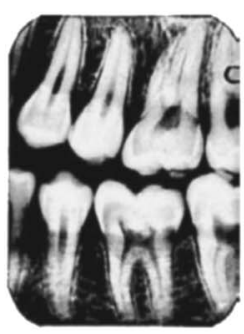

(b)

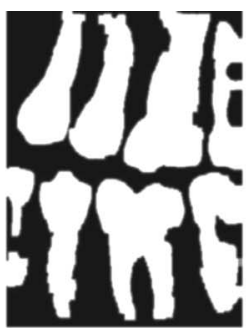

(c)

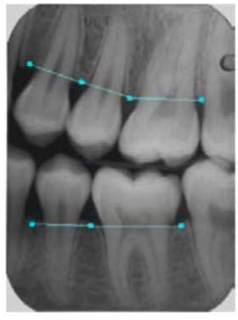

(d)

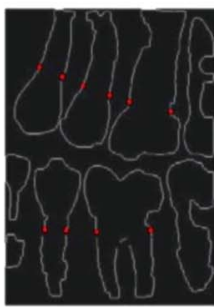

(e)

Fig. 4 Teeth segmentation: (a) original image, (b) result of top-hat enhancement, (c) morphological operation, (d) separated roots and crowns, and (e) final segmentation result.

bone and background areas can be suppressed as well. Figure 4(b) shows a result of the enhancement algorithm.

In bitewing segmentation, we use a window-based adaptive threshold to segment the teeth. The idea is to examine the intensity values of the local neighbors of each pixel. If the intensity value of the pixel is larger than the average intensity values of its neighbors, then it is classified as a tooth pixel, otherwise it is classified as background. To separate each tooth region, we apply a binary morphological operation to eliminate small noisy parts and smooth the tooth regions. Then, we subtract the tooth areas from the original image to obtain the bones and the background regions, and apply simple thresholding to separate the bones from the background. The results are shown in Figs. 4(b) and 4(c).

The positions of the bones provide information to approximate the gum line and separate the crown and root parts of the teeth. As shown in Fig. 4(d), the line that connects the tips of the bones approximates the gum line. To determine the gum lines we must separate the bones of the upper and the lower jaws and identify the tips of the bones. From the detected bones images, the upper and lower jaws are separated using integral projection. ${ }^{12}$ The bones between adjacent teeth are also separated using integral projection. Then we use the vertical integral projection analysis to find the orientation angle of each part of the jaw based on the bones image. We rotate the each of the two bones images in an angle range, say, $[-20,20]$, with a chosen interval, say, $1 \mathrm{deg}$. We establish the integral projection for each rotated image, assuming $N \times M$ is the size of the binary image, and $\alpha$ is its rotation according to original image, its integral projection function is

$\operatorname{hist}^{\partial}(i)=\sum_{k=1}^{N} f(k, i)$,

where $i$ is the $i$ 'th column. It is clear that the rotating angle of bones can be obtained by finding the rotated image with the $\alpha$ rotation, which has the maximal standard deviation of preseding function. The tips of the bones are then used to separate the crowns from the roots. As to the positions of these points on original dental image, we must rotate them back according to the orientation between the detected bones images and the original image. Figure 4(e) shows the final result of the segment stage. Figure 5 shows an example of another dental record and how it is segmented.

\subsubsection{Root and contour extraction for periapical images}

This methodology involves three stages radiograph segmentation: contour extraction and shape matching, as described in detail in Ref. 13. The radiograph segmentation stage utilizes the integral projection ${ }^{12}$ histogram in horizontal directions to find the gap between the mandibular and maxillary teeth (Fig. 6) and the integral projection histogram in vertical directions to find the gap between neighboring teeth (Fig. 7).

The contour extraction stage has two substages: crown shape extraction and root shape extraction. While detecting the crown contour, by assuming that the intensities of background pixels form the first mode in the intensity histogram, for a pixel with intensity $I$, we can find the probability for it to be a tooth pixel $p\left(\omega_{t} \mid I\right)$ and the probability for it to be a background pixel $p\left(\omega_{b} \mid I\right)$. Then, in each radial line through the center of the crown area, we find the most possible boundary point. The probability for a point $P$ to be a boundary point is defined as

$p_{B}(P)=p\left(\omega_{b} \mid I_{\text {outer }}\right) p\left(\omega_{t} \mid I_{\text {inner }}\right)$,

where $I_{\text {outer }}$ and $I_{\text {inner }}$ are the neighboring points of $P, P_{\text {outer }}$, and $P_{\text {inner}}$, as shown in Fig. 8.

The root shape detection is based on the two ends of the detected crown contour (Fig. 9). For the teeth in the lower

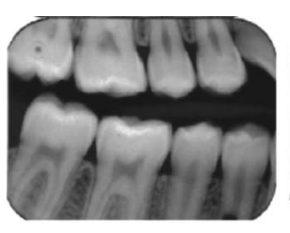

(a)

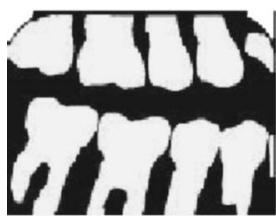

(d)

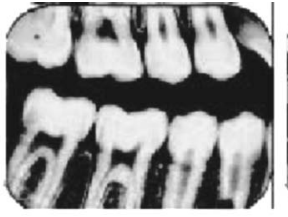

(b)

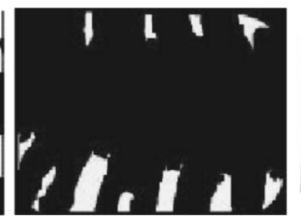

(e)

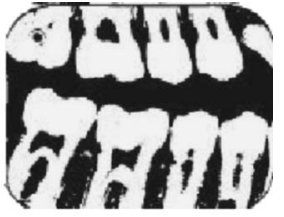

(c)

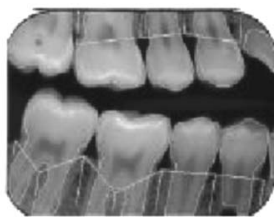

(f)
Fig. 5 Contour extraction for bitewing dental images. 

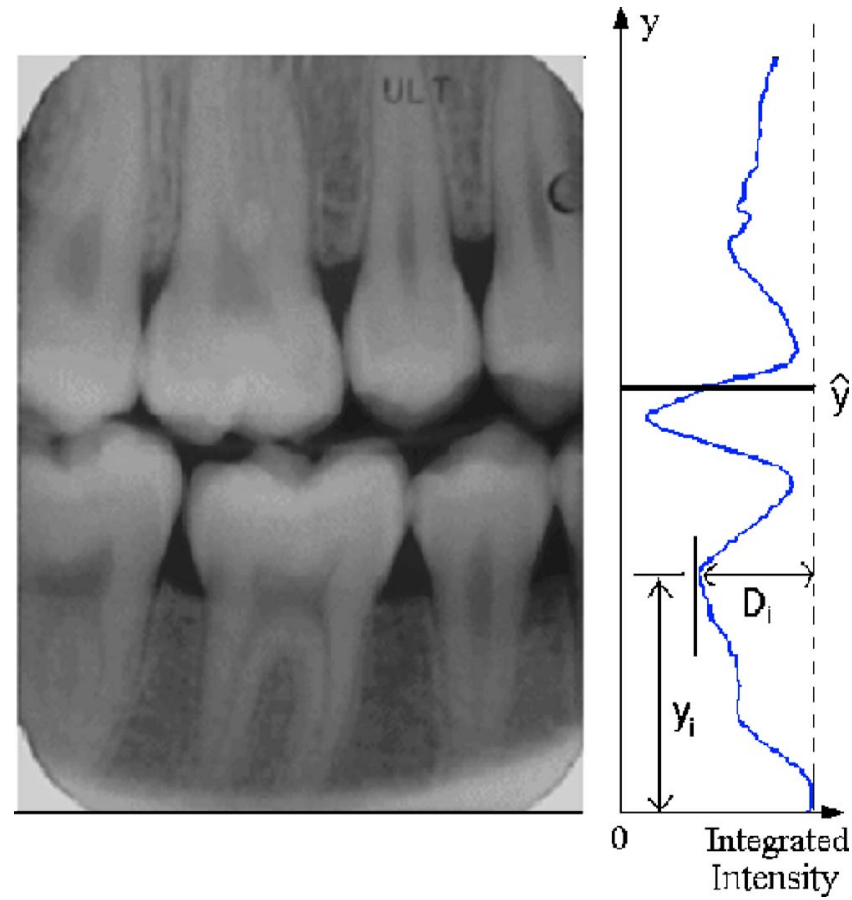

Fig. 6 Integral projection on the $y$ axis.

jaws, if the $i$ 'th point on the left/right root contour has coordinates $\left(x_{i}, y_{i}\right)$, the $i+1$ 'th point $\left(x_{i}+1, y_{i}+1\right)$ is computed iteratively as

$x_{i+1}=\arg \max _{x_{i}-r \leqslant x \leqslant x_{i}+r}\left(I_{\text {inner }}-I_{\text {outer }}\right)$,

$y_{i+1}=y_{i}+h$,

where $r$ is radius of the search space, and $h$ is the increment in the vertical position for each new point. The iteration ends when $y_{i}$ increases beyond the image boundary, or

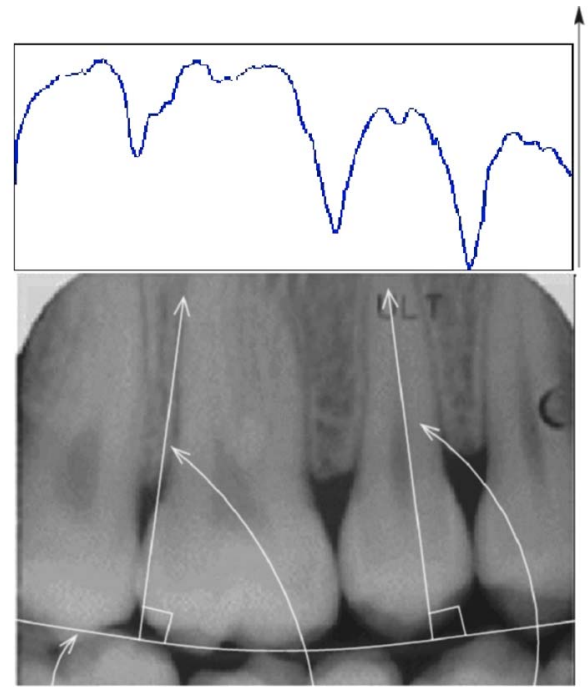

Integrated Intensity

\section{Curve sv}

Directions of Summing Up Pixcls

Fig. 7 Integral projection of pixels of the upper teeth along the lines perpendicular to the curve of the valley.

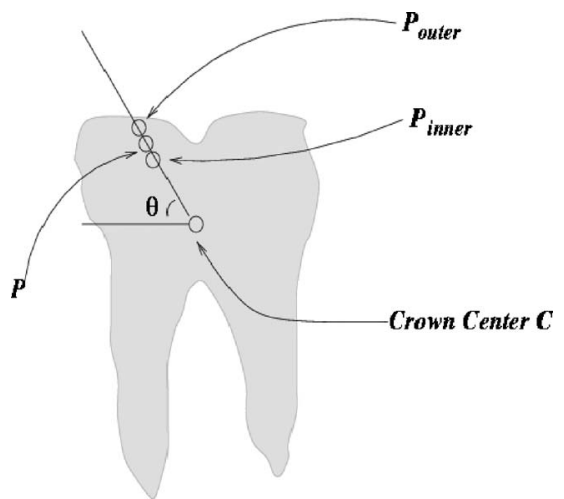

Fig. 8 Detection of crown shape.

$\max _{x_{i}-r \leqslant x \leqslant x_{i}+r}\left(I_{\text {inner }}-I_{\text {outer }}\right)$ is less than a threshold. Figures $10,11,12$, and 13 show examples of contour extraction for perapical dental images. ${ }^{13}$

In the shape matching stage, the shapes extracted from the query image must be matched to the shapes extracted from the database images. One of the main difficulties in matching AM and PM images is due to the fact that they were taken at different times often as long as several years. Thus, the viewpoints are usually slightly different, which causes an affine transformation between the two images that must be considered prior to shape fitting. We confine ourselves with a subclass of the affine transformation. In particular, we do not consider the shear transformation, because the shear is negligible in AM and PM images. Thus, the affine transformation $T$ is a function of the form

$T: R^{2} \rightarrow R^{2}, \quad T(P)=\mathbf{A} \cdot P+\tau$

where $P=(x, y)^{\mathrm{t}}$ represents a point in the query shape, $T(P)$ is the result of applying the transformation $T$ on $P, \mathbf{A}$ is the transformation matrix, and $\tau$ is the translation vector. The parameters in $\mathbf{A}$ and $\tau$ can be represented as

$\mathbf{A}=\left(\begin{array}{cc}\cos \theta & \sin \theta \\ -\sin \theta & \cos \theta\end{array}\right)\left(\begin{array}{cc}S_{x} & 0 \\ 0 & S_{y}\end{array}\right), \quad \tau=\left(\begin{array}{c}\tau_{x} \\ \tau_{y}\end{array}\right)$,

where $\tau$ is the rotation angle, $S_{x}$ and $S_{y}$ are vertical and horizontal scale factors, and $\tau_{x}$ and $\tau_{y}$ are vertical and horizontal translations. They are optimized for best fitting between the transformed query shape and the database shape.

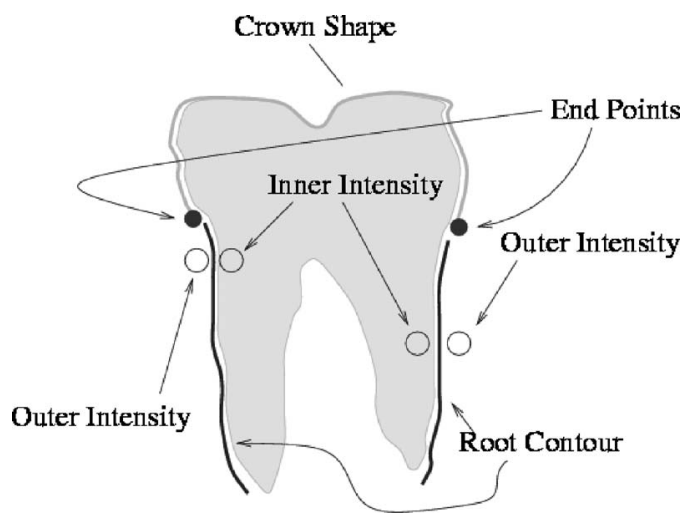

Fig. 9 Root shape detection. 


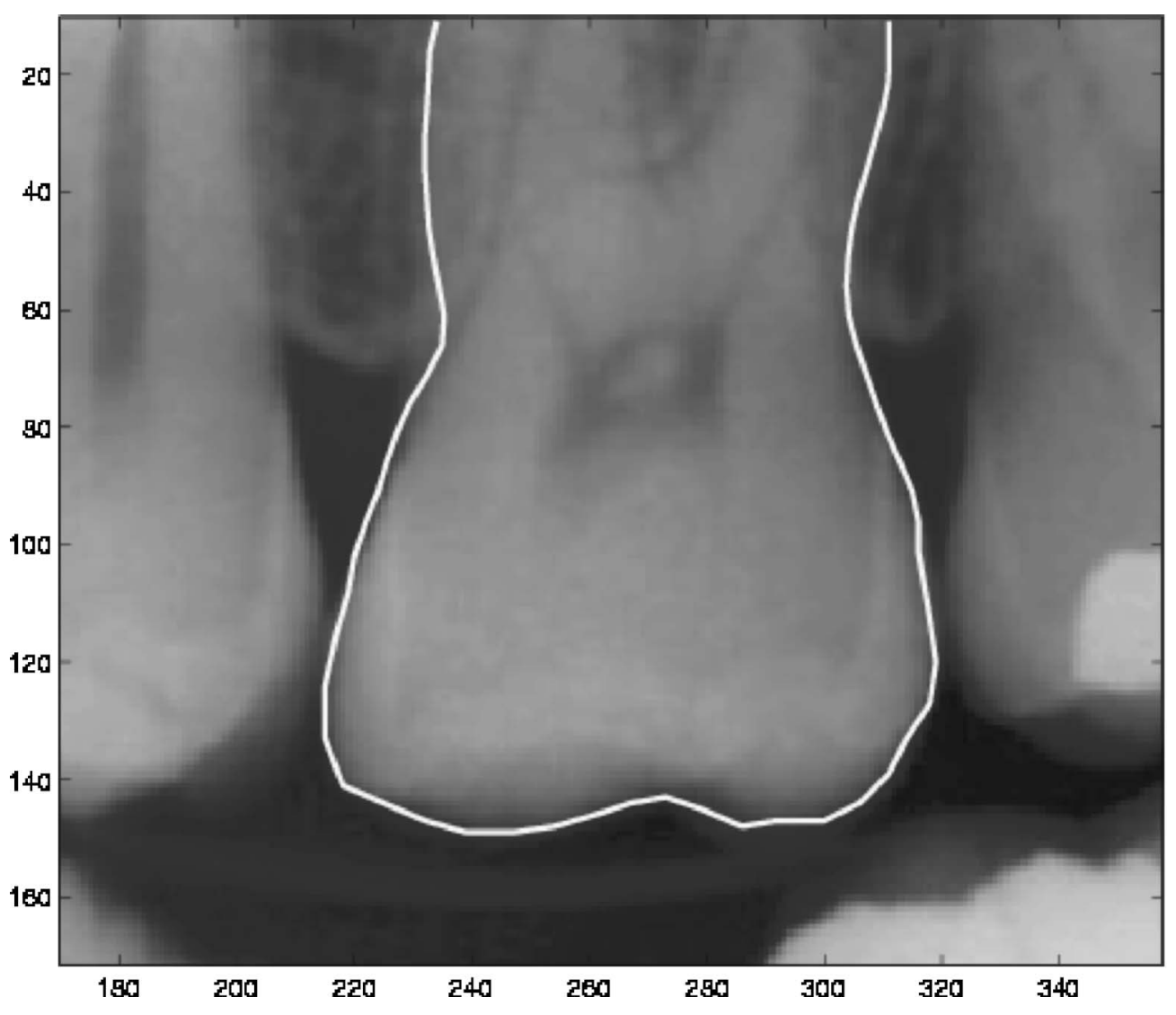

Fig. 10 Examples of contour extraction for perapical dental images. ${ }^{13}$

The measurement of fitting two radiographs is defined in this way. Given a query image, from every database image we generate several subimages, each containing the same number of teeth as the query image. The teeth in the query image and database subimages are labeled as $1,2, \ldots, 32$ from top right to top left to bottom left right bottom. Then teeth with the same label in query and database subimage form a pair. For each pair, we define the matching distance (MD) that must be minimized:

$$
\begin{aligned}
\operatorname{MD}(T) & =\sum_{\text {each pairs of shapes }} D(T), \\
D(T)= & \sum_{\mathrm{P}(\mathrm{i}) \in \text { query shape }}\left\{\min _{\mathrm{P}^{\prime}(\mathrm{j}) \in \text { database shape } \| P(i)}\right. \\
& \left.-T\left[P^{\prime}(j)\right] \|\right\} .
\end{aligned}
$$

So, $D(T)$ is the distance between a pair of teeth, and $\operatorname{MD}(T)$ is the summation of all $D(T)$ in a query. A candidate list is generated as the output, after the matching distances are sorted in the ascending order. Figure 14 shows an example of the matching stage. The black lines represent the teeth shapes in the AM images; the gray lines represent the query shapes after the transformation $T$. Note that in these examples, the matching distance for the genuine AM images is smaller than the matching distance for the imposter AM images.

\subsection{Image Comparison Matching Component}

This component takes the potential candidate list (that is, of the order of 100 to 150 records) provided from the first component and shrinks it down (to the order of 20 to 25 records) and delivers it to the forensic expert who makes the final decision about any possible matches.

In this component, the radiograph images are processed through three main stages: enhancement, segmentation, and matching. While the enhancement stage removes noise and enhances the quality of the radiograph images, the segmentation stage automatically segments the dental image into rectangular regions, where each region contains each tooth with its surroundings. These regions are then matched through a neural network based matcher as shown next.

\subsubsection{Enhancement}

Enhancement of a dental radiograph is the process of producing an improved quality image out of a degraded quality input image of a dental radiograph. The term "higher quality" is a fuzzy term that should be further explained. The quality of an image is a measure of its suitability for an application-specific manipulation. In ADIS, good-quality dental radiographs are those that would result in valid segments when used in conjunction with a suitable segmentation technique. Most segmentation techniques require high definition of object boundaries. From an image processing point of view, a digitized dental radiograph is an 8-bit grayscale image (at least for the dental image database provided 


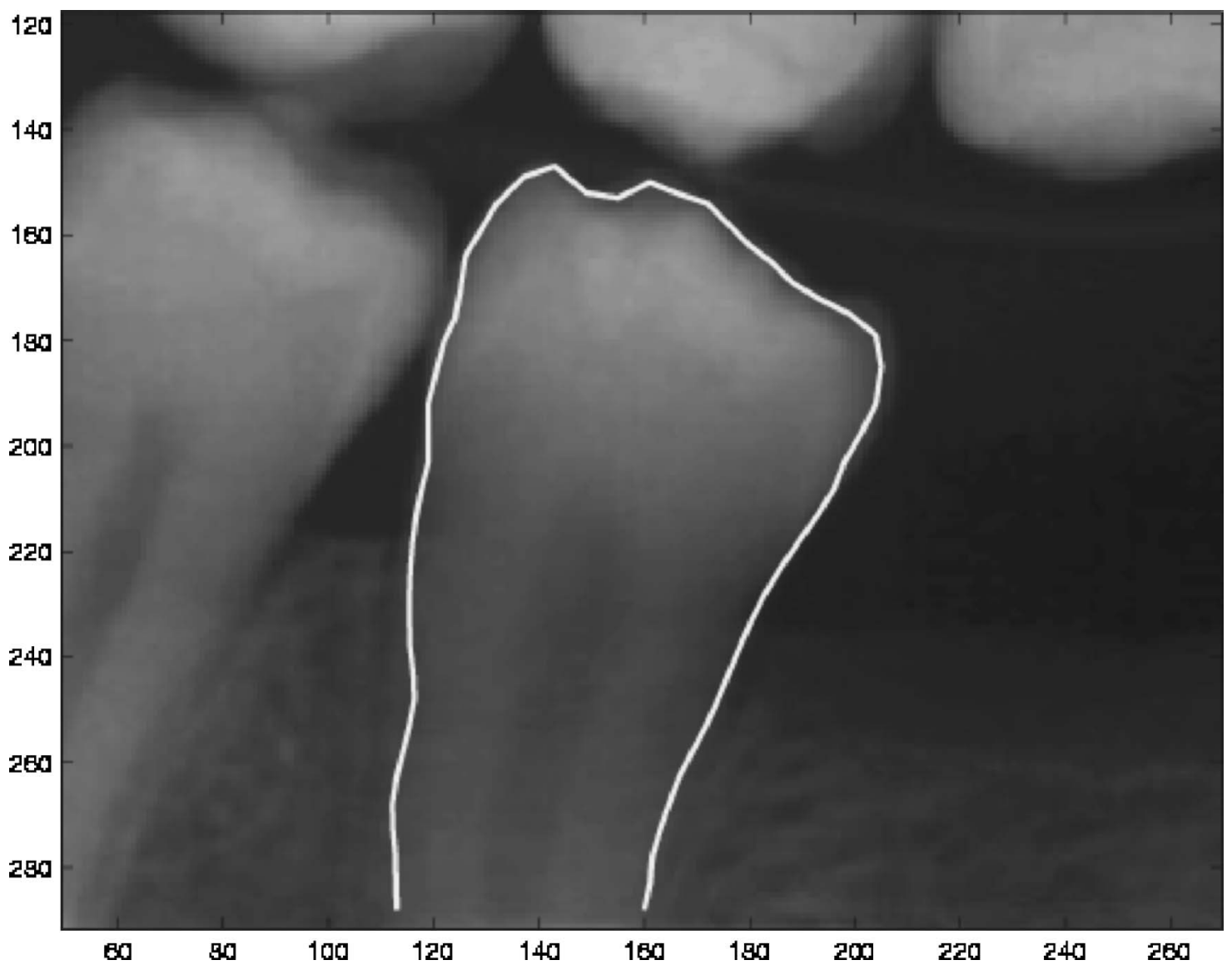

Fig. 11 Examples of contour extraction for perapical dental images. ${ }^{13}$

by the CJIS). The image size depends on the film type and the digitization resolution. Image resolution is characterized by the sampling rate (number of captured pixels per unit length) and the number of bits used in encoding the pixel colors (number of possible quantization levels). The CJIS database contains dental radiographs with various sampling rates.

In periapical and bite-wing views, we identify three main classes of "objects": teeth, gum, and air. A tooth maps to an area with mostly "bright" gray scales (except for the pulp tissue) while the gum maps to areas with "midrange" gray scales, and air maps to "dark" gray scales. Thus, a "significant" contrast in gray scales within a "small" area of a dental radiograph indicates a transition from one object to another. To assist segmentation, it is desirable to transform poor quality dental radiographs in a way that ensures an appreciable degree of contrast between the dominant gray scales used in capturing the different classes of objects. The enhancement transformation $\left(T_{\text {enh }}\right)$ in question maps a given gray scale $(g)$ from the input image to a new gray scale $T_{\text {enh }}(g)$ in the enhanced image. In fact, $T_{\text {enh }}$ is a discrete transformation, however, for ease of mathematical manipulation we will state some of its properties as if it was a continuous gray-scale enhancement transformation. We can describe $T_{\text {enh }}$ as: $T_{\text {enh }}(g):\left[g_{\min }, g_{\max }\right] \rightarrow[0,255]$ such that,

$$
\text { 1. } T_{\text {enh }}\left(g_{\text {min }}\right)=0 \text {, }
$$

2. $T_{\text {enh }}\left(g_{\text {max }}\right)=255$,

3. $T_{\text {enh }}^{\prime}(g)>0 \forall g \in\left(g_{\text {min }}, g_{\text {max }}\right)$,

4. $T_{\text {enh }}^{\prime}(g)>\gamma \forall g \in\left[g_{\text {th }}-\delta, g_{\text {th }}+\delta\right]$, and

5. $\Sigma H\left[T_{\text {enh }}(\mathrm{I})\right]<\Sigma H(I)$, where $\Sigma$ is taken over $g$ $\in\left[g_{\text {th }}-\delta, g_{\text {th }}+\delta\right]$,

where $g_{\min }$ is the index of the "darkest" gray scale in the input image, $g_{\max }$ is the index of the "brightest" gray scale in the input image, $g_{\text {th }}$ is the index of the "threshold" gray scale obtained from gray-scale histogram analysis of the input image, $\gamma$ is the minimum tangent slope of the transformation curve over the range of input gray scales specified in condition $4, \delta$ specifies the range of steep transition in the transformation curve, $H(-)$ is the gray-scale histogram of (-), and $I$ is the input image. Conditions 1 and 2 ensure that the darkest and the brightest gray scales in $I$ are mapped to "black" and "white", respectively. Condition 3 ensures that the transformation preserves the original relative order between gray scales. Condition 4 is meant to provide a steep transition in mapping gray scales in the neighborhood of $g_{\text {th }}$. Condition 5 guarantees that the contribution of the range of gray scales around $g_{\text {th }}$ decreases after enhancement.

The result of stretching the contrast of gray scales as just described would be an image with a gray-scale histogram that has more density of lines toward its edges, and less density of lines around the range of steep transition. In 


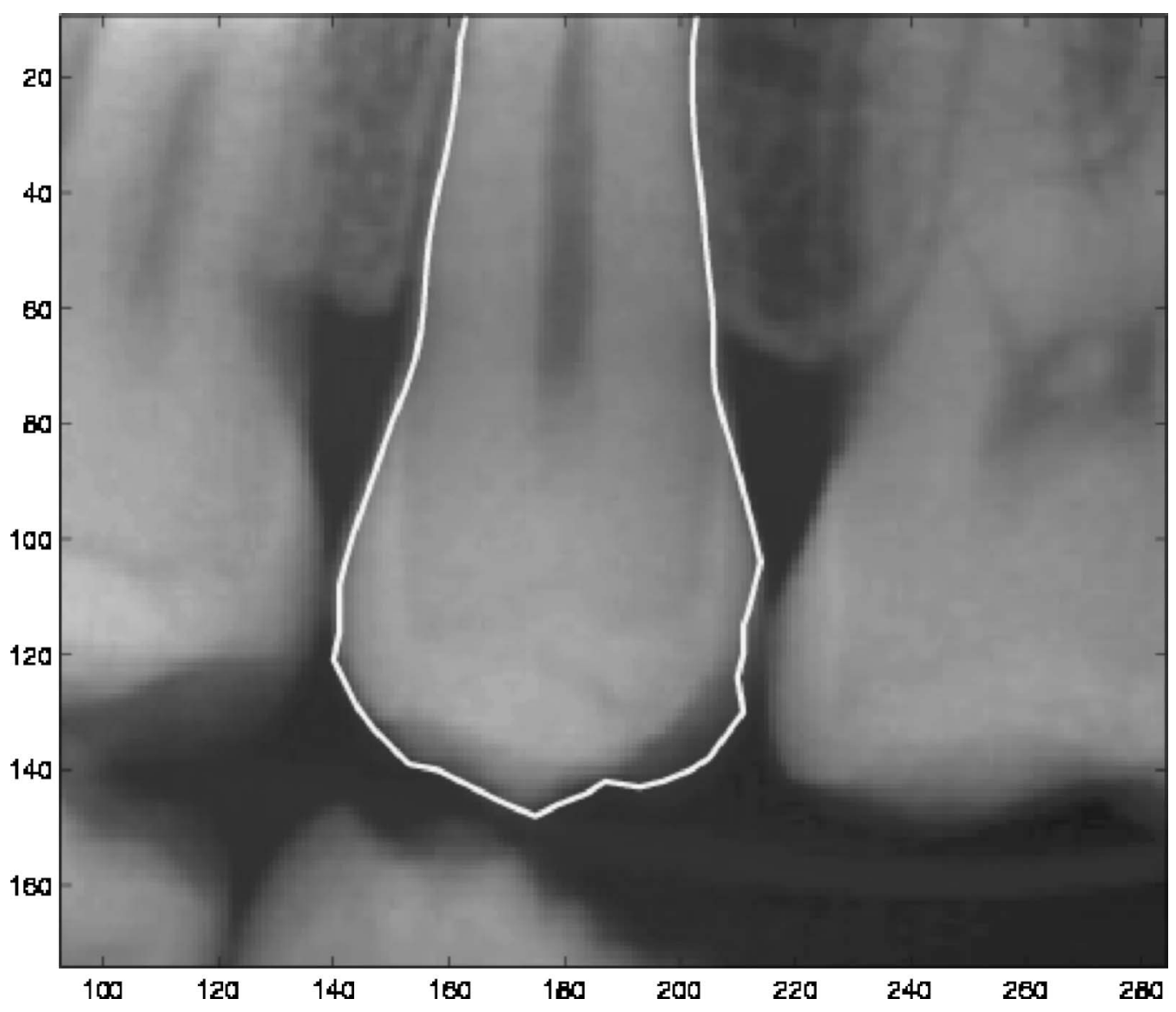

Fig. 12 Examples of contour extraction for perapical dental images. ${ }^{13}$

other words, the transformation tends to push the midrange gray levels toward either end, leaving less concentration of gray levels in the neighborhood of the transformed $g_{\text {th }}$.

Figure 15 shows an example of enhancing a periapical dental radiograph using our ADIC enhancment scheme. The original radiograph is shown in Fig. 15(a), the result after applying the enhancement transform is shown in Fig. 15(b). Note that tooth edges are emphasized. The grayscale histograms before enhancement and after enhancement are shown in Figs. 15(c) and 15(d), respectively. The solid vertical lines lines in Fig. 15(c) represent the computed marker gray scales and the dotted line represents a threshold gray scale used for adaptive enhancement.

\subsubsection{Segmentation}

In this stage, we consider only segmenting radiograph images into regions, where each region contains each individual tooth, rather than extracting the exact contour as in previous sections. This is due to the nature of the matcher that deals with regions and matches them with no need for the exact root or crown contour. We utilized the morphological filtering approach in this segmentation stages as described in Ref. 11. Figure 16 shows samples of our results with region-based morphological filtering segmentation.

\subsubsection{Matcher}

In this stage, we consider the decision-making stage of the ADIS prototype. The input to the decision-making stage is a pair of preprocessed representative segments from a candidate and a reference radiographs. The decision-making stage extracts embedded features from the input pair, and then determines their matching probability based on the measured differences between extracted features. As previously mentioned, this stage is composed of two layers: the feature extraction and the decision. The first layer applies a set of feature filters to the input pair, $A$ and $B$, each filter extracts a certain feature and thus transforms the input pair to an image pair in the corresponding feature space $(Z A, Z B)$. The differences between features $(\Delta Z)$ are computed then fed to a Bayesian decision-making layer, which uses the computed difference evidence $(\Delta Z)$ to estimate the posteriori probability of match $[p(M \mid \Delta Z)]$, as shown in Fig. 17. The parameters of both layers evolve by training with exemplar preprocessed pairs that are presented to the decision-making stage along with their matching status. The training process continues until a high accuracy in decision is observed. The feature extraction layer employs a set of filters to map an $n \times n$ pixel preprocessed dental image to a set of $m \times m$ pixel images in corresponding feature domains. This mapping is carried out by discrete spatial convolution using $w \times w$ pixel filter kernels. The kernel width and the overlap between image receptive fields are chosen such that the feature images capture the features presented in the preprocessed images while avoiding both over representation of the feature ( $\operatorname{small} w$ ) and under representation of the feature (large $w$ ). 


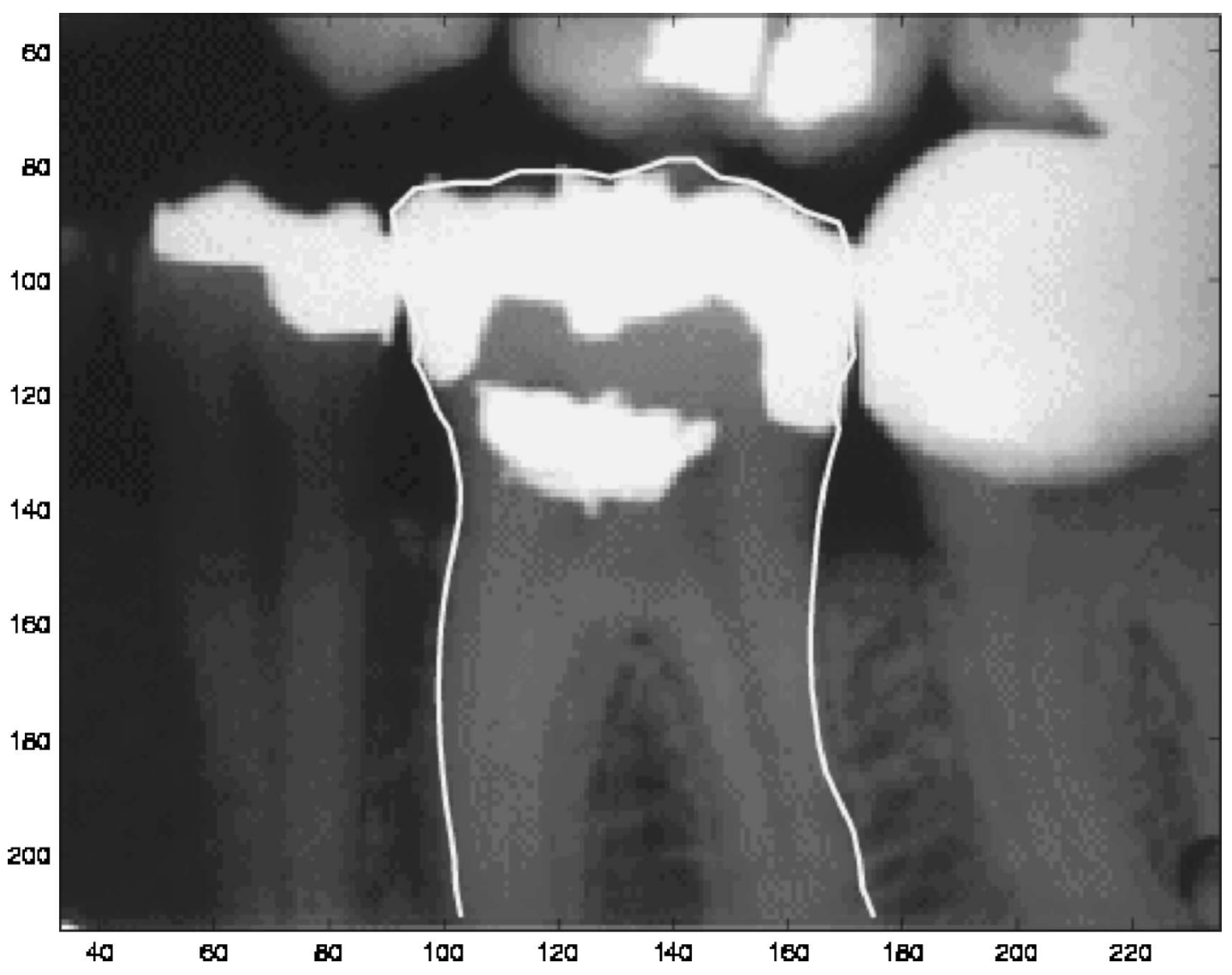

Fig. 13 Examples of contour extraction for perapical dental images. ${ }^{13}$

The decision layer is a binary Bayesian classifier with its two classes being a "matched" class and an "unmatched" class. The output of this layer is the probability that a submitted image pair belongs to the "matched" class $(M)$, which is $p(M \mid \Delta Z)$. The random variable $(\Delta Z)$ belongs to either the "matched" class or "unmatched" class, and its probability of occurrence is given as follows:
$p(\Delta Z)=p(\Delta Z \mid M) p(M)+p(\Delta Z \mid \bar{M}) p(\bar{M})$.

The question here is to find probability distribution functions (pdf) that best capture the relation between the random variable $\Delta Z$ and the posterior probability for either class. For the matched class case, such a pdf would be decreasing with $\Delta Z$; that is to say, the less the measured

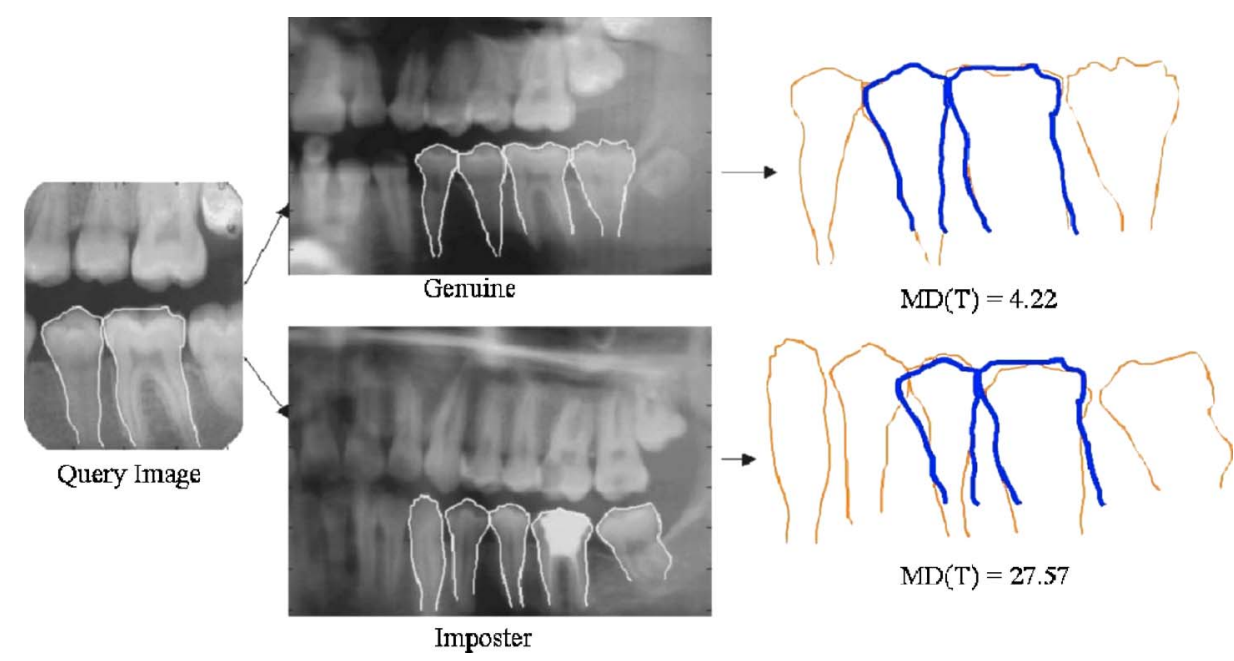

Fig. 14 Examples of the matching stage. 

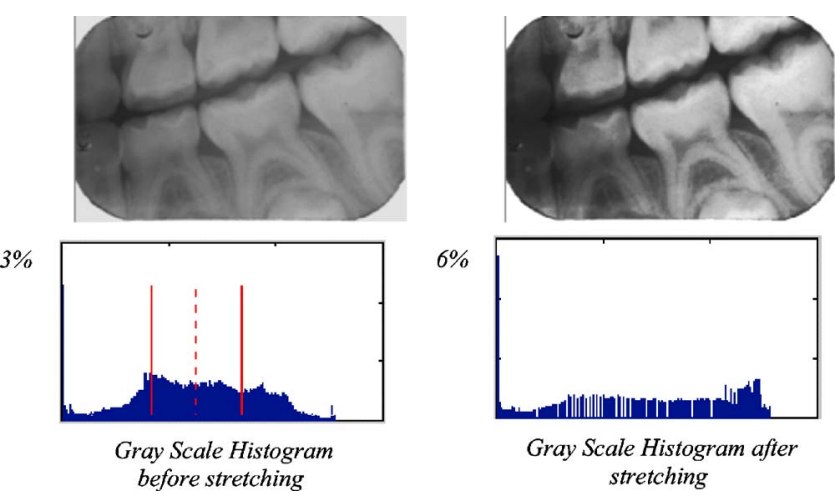

Fig. 15 ADIS image enhancement using gray-scale contrast stretching.

difference, the higher the probability that the pair of feature image patches belong to the matched class, and vice versa. Baldi and Chauvin suggested binomial pdf's, however, in case of dental images, we found that the geometric features require that we uniquely distinguish between the pdf's for the two classes. Thus, we added difference-weighting parameters to the binomial distribution. ${ }^{10}$

The match decision is made based on the value of the estimated match score, we use two thresholds that define three possible decisions: matched $[p(M \mid \Delta Z)>$ thr2], unmatched $[p(M \mid \Delta Z)<$ thr1], and undetermined [thr1 $<p(M \mid \Delta Z)<\mathrm{thr} 2]$. Choice of thr1 and thr2 values is made such that they provide good separation between the two classes, and that classification error meets the specifications in terms of the false rejection rate (FRR) and false acceptance rate (FAR).

System training is the process of presenting a set of exemplar images to the decision-making stage to guide the evolution of its parameters. Such a set of parameters should meet a certain specified accuracy in classifying the training set of dental radiographs. Generally, the training of a neural network is an optimization problem in which a cost function is to be minimized according to some constraints. In our case, the output of the decision-making stage is to be interpreted as a probability, and a suitable cost function would be the cross entropy between the estimated probability $p=[p(M \mid \Delta Z)]$ and the true probability assigned to an exemplar pair $P$, summed over the set of all training exemplars. The cross entropy function is a well-known information theoretic measure of discrepancy. ${ }^{14}$

Back propagation is the most well known technique for supervised training of neural networks. An important factor in achieving convergence in any gradient descent technique is choosing proper learning rates for the different weights. Moreover, for a given weight, it might be necessary to dynamically change the learning rate during the learning pro-

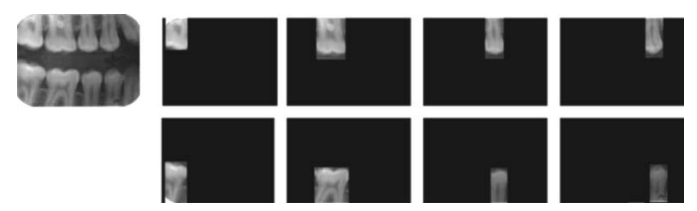

Fig. 16 Morphological filtering segment.

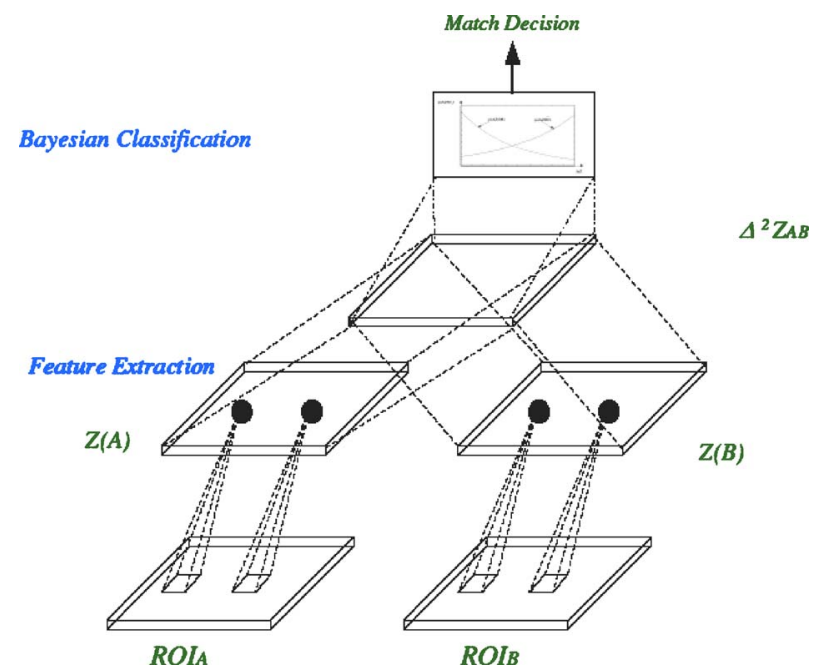

Fig. 17 Bayesian classifications.

cess so that convergence would be more probable. ${ }^{15}$ However, convergence is not guaranteed. Therefore, we explored use of a strategic searching technique to be used in conjunction with back propagation to insure fast convergence of system training.

We extended a univariation of parameters technique described in Ref. 15 so that it allows for more dynamics in the searching process, and to reduce its computational cost. Our technique manipulates each parameter individually as implied by "univariation." As any other training method, the target of our searching technique is to find a parameter set that minimizes the "known-to-be" convex cost function. In this technique, we propose that the cost function $(H)$ assumes quadratic dependence on each weight individually.

Considering one parameter at a time, we use a parameter's current value and two test values in its neighborhood $( \pm 10 \%$ of its current value). We compute the values of the cross-entropy function using the parameter's test values. Then, we apply quadratic interpolation of the three data points (the current point and the two test points). The coefficients of the resulting quadratic curve are used to anticipate the parameter's value corresponding to a minimum on the quadratic approximation of the cross-entropy function (we suggest that the cross-entropy function has a quadratic dependence on any of its parameter, when one parameter is studied at a time).

We use the obtained parameter value to determine the cross-entropy function, and check if this value truly causes the function to decrease. If so, the parameter value is affixed, otherwise the old value is restored. This process is carried out for each of the parameters (55 parameters per feature filter). Evidently, this technique is computationally more expensive than the simplest version of the backpropagation technique. Hence, we apply this adaptive technique when it is observed that back propagation does not seem to lead to a satisfactory minimum. Our hybrid training technique switches between back propagation and strategic searching based on observation of training improvement.

\section{Discussion, Conclusions, and Future Work}

We presented a prototype for an ADIS. ADIS can be used by law enforcement agencies for resolving cases of MUPs. 


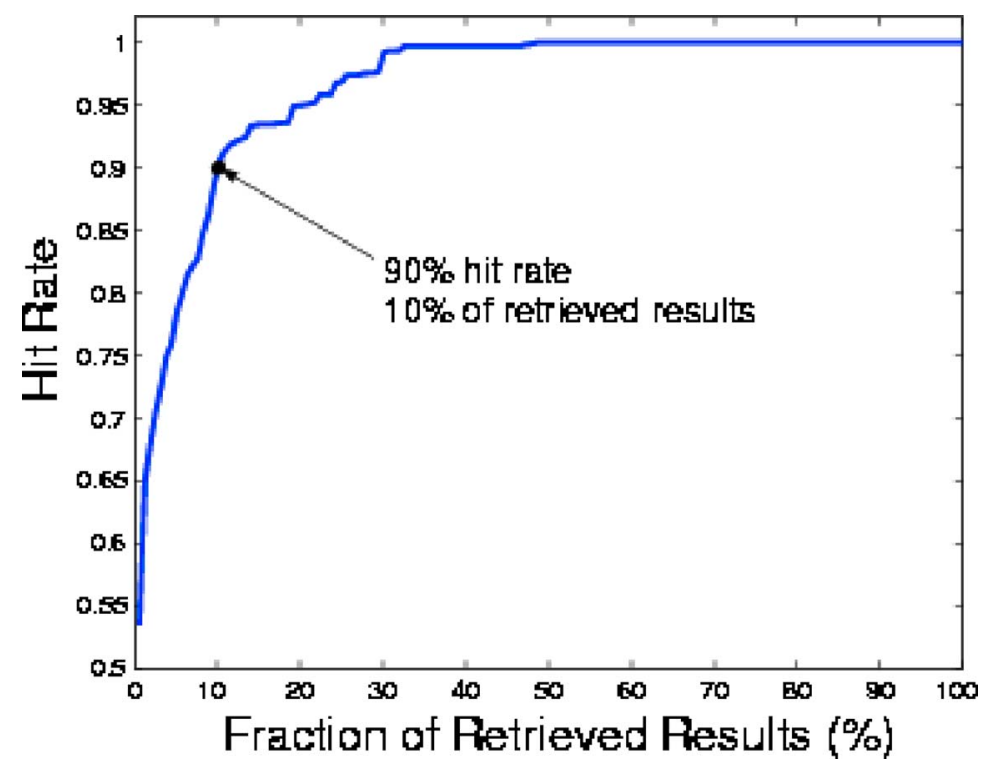

Fig. 18 Performance of the potential matching component.

The main contribution is the proposed ADIS architecture that can handle a large database of images. Our proposed architecture is split in two main stages, where the first stage reduces the number of candidate images from thousands to hundreds, and the other stage reduces the number of candidate images to a match list of a few cases to be given to the forensic expert for a final decision. Samples of the performance results of each part of this architecture are illustrated along with the description of the methodologies proposed. More extensive results of each component can be found in the reference of each methodology. This is the first attempt to present a comprehensive ADIS in the computer vision/ image processing and pattern recognition arena.

\subsection{Initial Testing of the Potential Matching Component}

Testing in Ref. 16 is conducted on a small database provided by FBI. We matched the 166 PM images to 235 AM images in the database. Experimental results show that there are $90 \%$ of cases for which the genuine images are placed among top $10 \%$ of retrieved images, as shown in Fig. 18. The potential matching component includes a tooth contour extraction technique adopted in Refs. 17 and 18 and a tooth labeling technique developed in Ref. 19.

\subsection{Initial Testing of the Image Comparison Component}

We tested the enhancement/segmentation performance of the preprocessing step and the decision-making step of the image comparison component. In a database of 1800 dental images, we achieved an enhancement/segmentation performance rate of $83 \%$. The matching accuracy of the decision making step, that is based on a neural network technique presented in Ref. 10, was found to be $82 \%$ with $8 \%$ FAR, $0 \%$ FRR, and $10 \%$ undetermined based on a test sample of 182 tooth pairs of AM/PM.

The main challenge to the current ADIS is the poor quality of several dental images. Other challenges occur when the subjects have much dental work (fillings etc), which limits the effectiveness of the proposed methodologies. Our future work will address several important problems such as the migration from region-based image matching to case-based image matching, processing poor quality radiographs, and effective and efficient retrieval from the DIR.

\section{Acknowledgments}

This research is supported in part by the U.S. National Science Foundation under Award No. EIA-0131079, the research is also supported under Award No. 2001-RC-CXK013 from the Office of Justice Programs, National Institute of Justice.

\section{References}

1. "Communique," The Canadian Dental Association (May-June 1997).

2. P. Stimson and C. Mertz, Forensic Dentistry, CRC Press, Boca Raton, FL (1997)

3. G. Gustafson, Forensic Odontology, American Elsevier, New York (1996).

4. American Society of Forensic Odontology, Forensic Odontol. News 16(2) (1997).

5. United States Army Institute of Dental Research Walter Reed Army Medical Center, "Computer Assisted Post Mortem Identification via Dental and other Characteristics," USAIDR Inf. Bull. 5(1) (1990).

6. J. McGivney et al., WinID2 software,www.winid.com.

7. N. R. Pal and S. K. Pal, "A review on image segmentation techniques," Pattern Recogn. 26(9), 1277-1294 (1993).

8. M. J. Smith and A. Docef, A Study Guide for Digital Image Processing, SP. Inc. (1999)

9. P. Maragos and R. W. Schafer, "Morphological systems for multidimensional signal processing," Proc. IEEE 78, 690-710 (Apr. 1990).

10. D. E. Nassar, "A prototype automatic dental identification system (ADIS)," MS Thesis, Department of Electrical and Computer Engineering, West Virginia University (Apr. 2001).

11. L. Vincent, "Morphological grayscale reconstruction in image analysis: application and efficient algorithms," IEEE Trans. Image Process. 2(2), 176-201 (1993).

12. R. Brunelli and T. Poggio, "Face recognition: features versus templates," IEEE Trans. Pattern Anal. Mach. Intell. 15(10), 1042-1052 (1993).

13. A. K. Jain and H. Chen, "Matching of dental X-ray images for human identification," Pattern Recogn. (submitted for publication).

14. P. Baldi and Y. Chauvin, "Neural networks for fingerprint image recognition," Neural Comput. 5 (1993).

15. C. Looney, Pattern Recognition Using Neural Networks, Oxford University Press (1997).

16. H. Chen and A. Jain, "Dental biometrics: alignment and matching of dental radiographs," in Proc. WACV, pp. 316-321, Breckenridge, CO 
(Jan. 2005).

17. O. Nomir and M. Abdel-Mottaleb, "A system for human identification from X-ray dental radiographs," Pattern Recogn. (in press)

18. J. Zhou and M. Abdel-Mottaleb, "A content-based system for human identification based on bitewing dental x-ray images," Pattern Recogn. (in press).

19. M. Mahoor and M. Abdel-Mottaleb, "Automatic classification of teeth in bitewing images," Pattern Recogn. (in press).

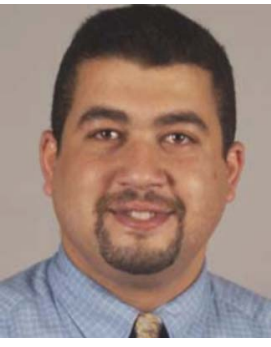

Gamal Fahmy received his BSc and MSc degrees from the University of Assiut, Egypt, in 1996 and 1998, respectively and his $\mathrm{PhD}$ from the Electrical Engineering Department, Arizona State University, in May 2003. His research interests include image compression/classification, image retrieval, biometrics, and characterizing visual data based on human perception. Dr. Fahmy is currently a research assistant professor with West Virginia University. He is working on multiple identification-technology-related projects funded by the Federal Bureau of Investigation, the National Institute of Justice, the Center of Identification Technology Research (CITeR), and the National Science Foundation.

Diaa E. M. Nassar is a computer engineering PhD student at West Virginia University. He received his BSc and MSc degrees in electrical engineering from Cairo University, Egypt, and West Virginia University in 1994 and 2001, respectively. He became a research assistant with West Virginia University in 1999. He has also worked in industry with Halliburton Company and Intel Corporation. His research interests are digital image processing, pattern recognition, soft computing, biometric systems, and quality assessment of software systems.

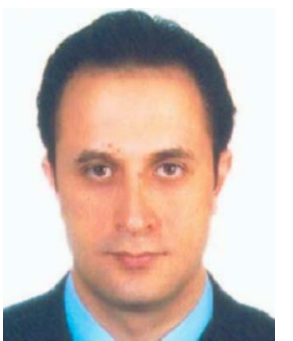

Eyad Haj-Said is a PhD student in the Lane Department of Computer Science and Electrical Engineering, West Virginia University (WVU). He is a member of the research assistant team in the Automated Dental Identification System (ADIS) project at WVU. He joined WVU in 2002. After receiving his MS degree in engineering and computer engineering from Stevens Institute of Technology, Eyad was a technical engineer with the Mankach Company from 1995 to 2000 and a teaching assistant with New York University from 2001 to 2002 .

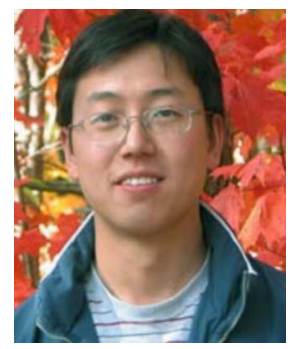

Hong Chen received his BSc and MSc degrees in computer science from Fudan University, Shanghai, China, in 1999 and 2002, respectively. He is currently working toward his PhD degree in the Department of Computer Science and Engineering, Michigan State University, Lansing. His research interests are pattern recognition, computer vision, and medical signal processing.

Omaima Nomir is a PhD student in the Department of Electrical and Computer Engineering, University of Miami, Florida. She received her BSc and MSc degrees from the Department of Computer Science and Automatic Control, Alexandria University, Egypt. Her research interests are pattern recognition, medical image processing, and neural networks.

Jindan Zhou received her BS and MS degrees in biomedical engineering from Southeast University, Nanjing, China, in 1999 and
2002. She is currently a PhD student in the Department of Electric and Computer Engineering of University of Miami, Florida. Her current research interests include image processing, pattern recognition, and biometrics.

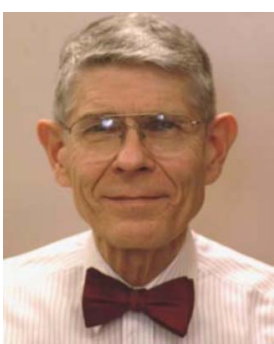

Robert Howell received his DDS degree from Virginia Commonwealth University, Medical College of Virginia, and his masters degree in oral pathology from Indiana University, Indianapolis. He is a professor of pathology with the West Virginia University School of Dentistry and a consultant to the West Virginia Northern Medical Examiner $\mathrm{FBI}$ Task Force on Dental Identification.

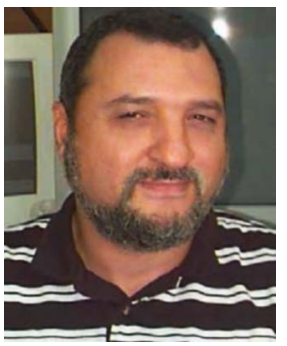

Hany H. Ammar is a professor of computer engineering with the Department of Computer Science and Electrical Engineering, West Virginia University. His research interests are identification technology and software engineering. Dr. Ammar has published over 100 papers in prestigious journals and conference proceedings. $\mathrm{He}$ served and is currently serving in the program and steering committees of several professional conferences and workshops.

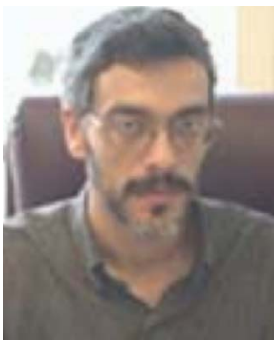

Mohamed Abdel-Mottaleb is an associate professor with the Department of Electrical and Computer Engineering, University of Miami. His research focuses on 3-D face recognition, dental biometrics, and human activity recognition. Prior to joining the University of Miami, from 1993 to 2000, he was with Philips Research, Briarcliff Manor, New York, where he was a principal member of the research staff and a project leader. There, he led projects in image processing and content-based multimedia retrieval. He holds 20 U.S. patents and has published over 60 papers in the areas of image processing, computer vision, and content-based retrieval.

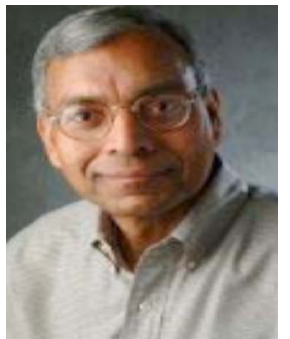

Anil K. Jain is a University Distinguished Professor with the Departments of Computer Science and Engineering at Michigan State University. His research interests include statistical pattern recognition, data clustering, and biometric authentication. $\mathrm{He}$ received awards for best papers in 1987 and 1991, and for outstanding contributions in 1976, 1979, 1992, 1997, and 1998 from the Pattern Recognition Society. He also received the 1996 IEEE Transactions on Neural Networks Outstanding Paper Award. He was the editor-in-chief of the IEEE Transactions on Pattern Analysis and Machine Intelligence from 1991 to 1994 . He is a fellow of the IEEE, $\mathrm{ACM}$, and the International Association of Pattern Recognition (IAPR). He has received a Fulbright Research Award, a Guggenheim fellowship, and the Alexander von Humboldt Research Award. He delivered the 2002 Pierre Devijver lecture sponsored by the IAPR. He holds six patents in the area of fingerprint matching. He is the author of a number of books, including the Handbook of Face Recognition (Springer, 2005), Biometric Systems: Technology, Design and Performance Evaluation (Springer, 2004), the Handbook of Fingerprint Recognition (Springer, 2003), and BIOMETRICS: Personal Identification in Networked Society (Kluwer, 1999). He is a member of the study team on Whither Biometrics being conducted by the National Academies. 